\section{Korrelationskoeffizient nach Pearson}

R.-D. Hilgers ${ }^{1}$, N. Heussen ${ }^{1}$ und S. Stanzel ${ }^{2}$

${ }^{1}$ Institut für Medizinische Statistik, Universitätsklinikum der RWTH Aachen, Aachen, Deutschland

${ }^{2}$ Heidelberg, Deutschland

Synonym(e) Korrelationskoeffizient nach Bravais-Pearson; Pearson-Korrelationskoeffizient; Pearson'scher Korrelationskoeffizient; Produkt-Moment-Korrelationskoeffizient

Englischer Begriff Pearson's correlation coefficient

Definition Der Korrelationskoeffizient nach Pearson (Pearson-Korrelationskoeffizient) ist ein quantitatives $\mathrm{Ma} ß$ zur Beurteilung der Stärke der Beziehung zwischen 2 stetigen Merkmalen (s. $\triangleright$ Merkmal). Er beschreibt die lineare Komponente des Zusammenhangs zwischen den beiden Merkmalen.

Beschreibung Der Pearson-Korrelationskoeffizient ist eine normierte Maßzahl und nimmt Werte zwischen -1 und +1 an. Ein Wert von +1 zeigt einen exakt positiv linearen Zusammenhang zwischen den Ausprägungen der beiden Merkmale an, während ein Wert von -1 im Falle eines exakt negativ linearen Zusammenhangs auftritt.
Je stärker der positive bzw. negative lineare Zusammenhang zwischen den Ausprägungen der beiden Merkmale ist, umso näher wird der Wert bei +1 bzw. -1 liegen. Ist kein linearer Zusammenhang zwischen den Ausprägungen der beiden Merkmale nachzuweisen, nimmt der Pearson-Korrelationskoeffizient einen Wert nahe Null an. Der Pearson-Korrelationskoeffizient zwischen 2 Merkmalen X und Y steht in engem Zusammenhang zum Regressionskoeffizienten ( $\triangleright$ Regressionskoeffizient) einer linearen Regression ( $\triangleright$ Regression, lineare):

Dividiert man das Produkt aus Pearson-Korrelationskoeffizienten und $>$ Varianz der y-Werte durch die Varianz der $\mathrm{x}$-Werte so ergibt sich ein $>$ Schätzer für den Regressionskoeffizienten. Damit lässt sich aus dem Pearson-Korrelationskoeffizienten der Regressionskoeffizient schätzen und umgekehrt. Als Maß für die Güte der Anpassung, die eine Regression erzielt, dient das sog. Bestimmtheitsmaß, das sich als Verhältnis der Varianz der geschätzten Werte zur Varianz der beobachteten Werte ergibt. Speziell bei der einfachen linearen Regression ist das Bestimmtheitsmaß identisch mit dem Quadrat des Pearson'schen Korrelationskoeffizienten.

\section{Literatur}

Hartung J, Elpelt B, Klösener KH (1995) Statistik - Lehr- und Handbuch der angewandten Statistik. Oldenbourg Verlag, München 